\title{
Gas-kinetic unified algorithm for computable modeling of Boltzmann equation and application to aerothermodynamics for falling disintegration of uncontrolled Tiangong- No.1 spacecraft
}

\author{
Zhi-Hui Li $\mathrm{i}^{1,2^{*}}$ (D) Ao-Ping Peng ${ }^{1}$, Qiang Ma ${ }^{1,3}$, Lei-Ning Dang ${ }^{1}$, Xiao-Wei Tang ${ }^{1}$ and Xue-Zhou Sun ${ }^{1,2}$
}

\footnotetext{
* Correspondence: zhli0097@x263. net

${ }^{1}$ Hypervelocity Aerodynamics Institute, China Aerodynamics Research and Development Center, P.O.Box 211, Mianyang 621000, China

${ }^{2}$ National Laboratory for Computational Fluid Dynamics, BUAA, No.37 Xueyuan Road, Beijing 100191, China

Full list of author information is available at the end of the article
}

\begin{abstract}
How to solve the hypersonic aerothermodynamics around large-scale uncontrolled spacecraft during falling disintegrated process from outer space to earth, is the key to resolve the problems of the uncontrolled Tiangong-No.1 spacecraft reentry crash. To study aerodynamics of spacecraft reentry covering various flow regimes, a Gas-Kinetic Unified Algorithm (GKUA) has been presented by computable modeling of the collision integral of the Boltzmann equation over tens of years. On this basis, the rotational and vibrational energy modes are considered as the independent variables of the gas molecular velocity distribution function, a kind of Boltzmann model equation involving in internal energy excitation is presented by decomposing the collision term of the Boltzmann equation into elastic and inelastic collision terms. Then, the gas-kinetic numerical scheme is constructed to capture the time evolution of the discretized velocity distribution functions by developing the discrete velocity ordinate method and numerical quadrature technique. The unified algorithm of the Boltzmann model equation involving thermodynamics non-equilibrium effect is presented for the whole range of flow regimes. The gas-kinetic massive parallel computing strategy is developed to solve the hypersonic aerothermodynamics with the processor cores 500 45,000 at least 80\% parallel efficiency. To validate the accuracy of the GKUA, the hypersonic flows are simulated including the reentry Tiangong-1 spacecraft shape with the wide range of Knudsen numbers of 220 0.00005 by the comparison of the related results from the DSMC and N-S coupled methods, and the low-density tunnel experiment etc. For uncontrolling spacecraft falling problem, the finite-element algorithm for dynamic thermalforce coupling response is presented, and the unified simulation of the thermal structural response and the hypersonic flow field is tested on the Tiangong- 1 shape under reentry aerodynamic environment. Then, the forecasting analysis platform of end-of-life largescale spacecraft flying track is established on the basis of ballistic computation combined with reentry aerothermodynamics and deformation failure/disintegration.
\end{abstract}

Keywords: Aerodynamics covering all flow regimes, Boltzmann model equation in thermodynamic non-equilibrium effect, Gas-Kinetic Unified Algorithm, Simulation of structural failure/ disintegration, Numerical forecast of flying path 


\section{Introduction}

Large-scale spacecraft in low orbit of $300 \mathrm{~km} \sim 500 \mathrm{~km}$ faces the problems of de-orbiting fall around the end of life, and disintegrates during reentering back to the earth because they suffer tremendous aerothermodynamics environment and overloads [1]. The high-temperature thermo-chemical non-equilibrium gas flows produced by spacecraft re-entering atmospheric surrounding will create cumulative effect on the metal truss softening, melting and the pyrolysis ablation of embedded composite. Under this circumstance, hypersonic aerothermodynamics problems need to be solved and meanwhile various fluid-structure interaction approaches are studied [2]. Aerothermodynamics induces structural deformation, softening, melting, ablation and disintegration. Figure 1 shows the schematic diagram of disintegrated flight path during reentry of large-scale spacecraft fall from the outer space. How to forecast the flight trajectory of uncontrolled spacecraft, disintegrated components and debris ahead of time is of world problem in the field of spaceflight. The precision of reentry forecast for disintegrated flight path mainly depends on the integrated simulation of aerothermodynamics and structural deformation failure/disintegration covering various flow regimes [3-7].

To simulate the gas flows from various regimes, the traditional way is to deal with them with different methods. On the one hand, the methods related to rarefied gas flow have been developed, such as the microscopic molecular-based Direct Simulation Monte-Carlo (DSMC) method $[8,9]$. On the other hand, also the methods adapted to continuum flows have been well developed, such as the Navier-Stokes equation solvers of macroscopic fluid dynamics. However, both methods are totally different in nature, and the computational results are difficult to link up smoothly with various flow regimes. Engineering development of current or intending spaceflight projects is closely concerned with complex gas dynamic problems of low-density flows in the intermediate range of Knudsen numbers, especially in the rarefied transition and in the near-continuum flow regimes. In fact, the Boltzmann equation [10] depicts the evolutionary process of the molecular velocity distribution function (VDF) from non-equilibrium to equilibrium state at arbitrary time in gases. It can describe the molecular transport phenomenon covering continuum flow to free-molecular flow regimes. The Navier-Stokes equation based on continuum medium assumption can be obtained according to the first-order Chapman-Enskog expansion of the Boltzmann equation. And one can prove that, for simple gases, when the number of simulated molecules approaches to infinite, the solution of the DSMC method, which has been widely using in rarefied gas dynamics, will be converged to that of the Boltzmann equation $[11,12]$. Due to the complexity and uncertainties $[10,13]$ of the nonlinear multidimensional integral-differential properties of the Boltzmann equation, it is very difficult directly and exactly to solve the Boltzmann equation.

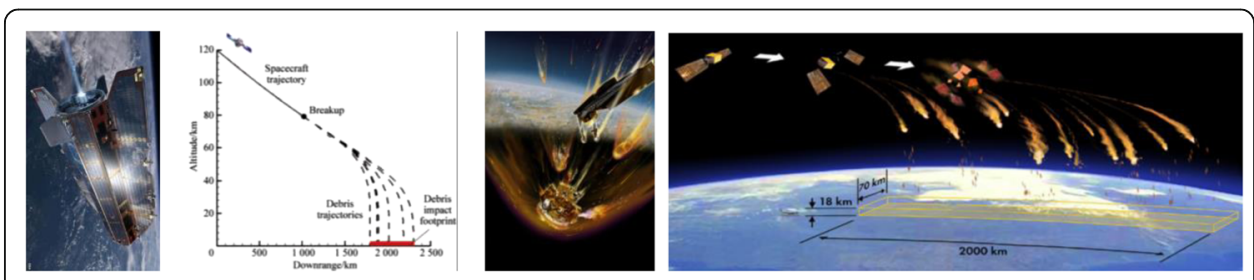

Fig. 1 Sketch of disintegrated flight path during reentry of large-scale spacecraft in expiry of service 
To take full advantage of collision relaxation and transport characteristics of the Boltzmann equation, several approximate solution methods have been developed commendably, such as the linearized Boltzmann equation [14, 15], moment method [16], Monte-Carlo finite difference method [17], and model equation methods [18-21]. In these techniques, the model equation methods have been widely used due to its simple frame and common numerical techniques [22-25]. By using a simplified collision relaxation model to replace the collisional integral of the Boltzmann equation, the kinetic model equation retains the basic properties of the Boltzmann equation, such as the $\mathrm{H}$-theorem and conservation invariant conditions. The most famous kinetic model is the BGK model [18] for monatomic gases. However, the BGK model leads to the Prandtl number $\operatorname{Pr}=1$, while its correct value is about $2 / 3$ for monatomic gases. In order to get correct Pr, some modified model equations such as the ES model by Holway [19] and the Shakhov model [20] were introduced. During the last two decades, the Lattice Boltzmann Method (LBM) [26, 27], the KFVS [28], a series of gas kinetic BGK-type schemes and direct modeling methods such as GKS, UGKS etc. [29-34], have been developed.

To study aerodynamic problems covering various flow regimes, the unified computable modeling has been actualized on the collision integral of the Boltzmann equation, in which the molecular collision relaxing parameter and the local equilibrium distribution function can be integrated with the macroscopic flow variables, the gas viscosity transport coefficient, the thermodynamic effect, the molecular power law, molecular models, and the flow state controlling parameter from various flow regimes [24, 35-38], and the gas-kinetic unified algorithm (GKUA) has been presented and used to simulate the gas flows from highly rarefied free-molecular flow to continuum flow regimes with the whole range of Knudsen numbers [37-39]. In the past, the GKUA has been successfully applied to solve hypersonic reentry aerothermodynamics around kinds of space vehicles and micro-scale flows involved in MEMS devices [6, 35-40]. Because the VDF $(f)$ is a multi-space and multi-dimensional function on velocity space and physical space, especially for the three-dimensional Boltzmann model equation, which is a six-dimensional $\left(x, y, z, V_{x}, V_{y}, V_{z}\right)$ function, large computational memory is consumed while directly solving the kinetic model equation, and it is very important how to improve the efficiency of numerical computation for three-dimensional hypersonic flows around complex bodies. The computational time step of explicit schemes is determined by the stability conditions of numerical schemes and would be very small for hypersonic flows around complex irregular vehicles due to metric coefficients of grid system and singularity of irregular objects, especially at continuum and near continuum flow regimes of near-space flying surroundings. So, it is necessary to construct the gas-kinetic implicit schemes $[6,7]$ in order to shorten the computing convergence time and improve the computational efficiency.

On the other hand, to simulate the thermal response destructive behavior, a finite element algorithm (FEA) has been developed [5] for the dynamic thermo-elasticity coupling problem of materials incorporated with the GKUA for solving the exterior hypersonic reentry aerothermodynamic environment, in which the Newmark implicit scheme is employed to discretize the dynamic thermoelasticity equation and the Crank-Nicolson scheme is used to solve the heat-conduction equation [41]. In engineering problems, the determination of thermal stresses is usually carried out in two steps. First, the temperature distribution is obtained from the Fourier's heat-conduction equation, the stresses are then calculated by the equations of thermoelasticity, including 
the temperature terms in the stress-strain relations. When subjected to the strong aerodynamic force and rigorous aerodynamic heating, the material deformation will significantly affect the temperature distribution in the interior of the material. The thermal-induced vibration should also not be neglected, and the heat propagation should be viewed as a wave phenomenon rather than a diffusion phenomenon. For this reason, a unified consideration and analysis to the coupled system of heat transfer, deformation and stresses on material and structure [42] is needed to simulate the real physical and mechanical problem. Thus, the dynamic thermo-elasticity coupling response behaviors including material internal temperature distribution, structural deformation, and thermal damage are simulated in real time by applying the FEA + GKUA in the field of reentering hypersonic aerothermodynamics.

It is key and necessary to solve multi-body flow interference once spacecraft disintegration is appeared during its falling and reentering back to the earth. The LU-SGS implicit scheme and the cell-centered finite volume method are constructed to solve the Boltzmann model equation under the frame of the GKUA. In this work, to solve the multi-body aerodynamic problems including two and three side-by-side cylinders and irregular bodies with different gap ratio covering highly rarefied to near-continuum flow regimes, a multi-block patched grid technique is built for irregular multi-body flows, in which the grid points on both sides of joint interface are completely patched by one to one, and the discrete distribution functions and macroscopic flow variables for computation are transferred by the interfaces between blocks. On the other hand, the GKUA will be further extended and developed to solve the thermodynamic non-equilibrium hypersonic flows during the re-entry disintegration of end-of-life spacecraft, in which a kind of Boltzmann model equation considering the excitation of vibrational energy is constructed.

To establish the forecasting platform of flying track for the uncontrolled spacecraft falling from outer space to earth as the first attempt, the DSMC for hypersonic reentry thermochemical non-equilibrium flow, N-S/DSMC, slip N-S as verifying tools, and the computational methods of thermal environment and structural heat transfer/composite material pyrolysis, and disassembly and separation have been developed [7, 9, 43, 44] by taking the coupling simulation with trajectory and aerothermodynamic calculation as the main line combined with statistical analysis and 3D scene visualization, the forecasting analysis platform of flying track for the end-of-life large-scale spacecraft is established for the unified computation of reentry aerothermodynamics, deformation failure/ablation/disintegration with engineering treatment. The remaining parts of this paper are organized as follows: The unified Boltzmann model equations in thermodynamic non-equilibrium effect and implicit numerical scheme are constructed in Section 2. The dynamic coupled thermo-elasticity equations and the finite-element algorithm are proposed in Section 3. The unified simulation of structural deformation and hypersonic aerothermodynamics is presented in Section 4. The numerical simulation and analysis of aerothermodynamics for disintegrated spacecraft is in Section 5, followed by conclusions and the expectation of the future work in Section 6.

\section{Gas-kinetic unified algorithm for thermodynamic non-equilibrium Boltzmann model equation}

The Boltzmann equation [10] depicts the evolutionary process of the VDF from non-equilibrium to equilibrium state at arbitrary time in monatomic gas. It connects the microscopic molecular dynamics and the macroscopic fluid mechanics by the 
probability statistical distribution function, and can describe the transport phenomena covering continuum flow to free-molecular flow regimes. Gas flow approaches to steady state and obeys the fact that the VDF goes to the local equilibrium distribution taken by macroscopic flow variables in the locality. So, the computable modelling of the Boltzmann equation can be realized $[6,24,35-40,45]$ by using the gas molecular collision relaxing parameter and the local equilibrium distribution function. Based on the processing mode of continuous energy level, the rotational and vibrational energy in the quantum states can be introduced as the independent variables of VDF. The equilibrium distribution functions involving in the energy modes and the effective temperature of translation \& rotation, or translation, rotation \& vibration can be deduced in computable forms, and the three-temperature unified Boltzmann model equation in non-equilibrium effect with vibrational energy is presented in the framework of GKUA, in which the relaxation process of gas molecules is simplified as translation, translation-rotation, and translation-rotation-vibration energy relaxation.

$$
\frac{\partial f_{i}}{\partial t}+\vec{V} \cdot \frac{\partial f_{i}}{\partial \vec{r}}=v_{t o t}\left[\begin{array}{l}
\left(1-\frac{1}{Z_{r o t}}-\frac{1}{Z_{v i b}}\right)\left(f_{i}^{(t)}-f_{i}\right)+\frac{1}{Z_{r o t}}\left(f_{i}^{(t, r)}-f_{i}\right) \\
+\frac{1}{Z_{v i b}}\left(f_{i}^{(t, r, v)}-f_{i}\right)
\end{array}\right]
$$

Where, $i=1,2$, 3, and,

$$
\begin{aligned}
& f_{1}^{(t)}=n\left(\frac{m}{2 \pi k_{B} T_{t r}}\right)^{3 / 2} \exp \left(-\frac{m C^{2}}{2 k_{B} T_{t r}}\right), f_{2}^{(t)}=\frac{\delta_{r o t} k_{B} T_{r o t}}{2} f_{1}^{(t)}, f_{3}^{(t)}=\frac{\delta_{v i b}\left(T_{v i b}\right) k_{B} T_{v i b}}{2} f_{1}^{(t)}, \\
& f_{1}^{(t, r)}=n\left(\frac{m}{2 \pi k_{B} T^{r}}\right)^{3 / 2} \exp \left(-\frac{m C^{2}}{2 k_{B} T^{r}}\right), f_{2}^{(t, r)}=\frac{\delta_{r o t} k_{B} T^{r}}{2} f_{1}^{(t, r)}, f_{3}^{(t, r)}=\frac{\delta_{v i b}\left(T_{v i b}\right) k_{B} T_{v i b}}{2} f_{1}^{(t, r)} \\
& f_{1}^{(t, r, v)}=n\left(\frac{m}{2 \pi k_{B} T_{o v}}\right)^{3 / 2} \exp \left(-\frac{m C^{2}}{2 k_{B} T_{o v}}\right), f_{2}^{(t, r, v)}=\frac{\delta_{r o t} k_{B} T_{o v}}{2} f_{1}^{(t, r, v)}, f_{3}^{(t, r, v)}=\frac{\delta_{v i b}\left(T_{o v}\right) k_{B} T_{o v}}{2} f_{1}^{(t, r, v)} . \\
& v_{t o t}=\operatorname{Pr} \cdot \frac{2 \alpha(5-2 \omega)(7-2 \omega)}{5(\alpha+1)(\alpha+2) \sqrt{\pi}} \cdot \frac{n T_{t r}^{1-X}}{K n_{\infty}} \cdot \frac{1}{Z_{i}} .
\end{aligned}
$$

All flow variables are evaluated and updated by three reduced non-equilibrium VDFs of $f_{1}, f_{2}$ and $f_{3}$ over the velocity space.

$$
\begin{aligned}
& n(\vec{r}, t)=\int f_{1}(\vec{r}, \vec{V}, t) d \vec{V}, \\
& n \vec{U}_{i}(\vec{r}, t)=\int \vec{V}_{i} f_{1}(\vec{r}, \vec{V}, t) d \vec{V}, \\
& \frac{3 \mathrm{n}}{2} T_{t r}(\vec{r}, t)=\int c^{2} f_{1}(\vec{r}, \vec{V}, t) d \vec{V}, \\
& \frac{\delta_{r o t}}{2} T_{t r}(\vec{r}, t)=\frac{1}{n} \int f_{2}(\vec{r}, \vec{V}, t) d \vec{V}, \\
& \frac{\delta_{\text {vib }}}{2} T_{\text {vib }}(\vec{r}, t)=\frac{1}{n} \int f_{3}(\vec{r}, \vec{V}, t) d \vec{V} \\
& p(\vec{r}, t)=n(\vec{r}, t) k T(\vec{r}, t), \\
& \tau_{i j}=2 \int c_{i} c_{j} f_{1}(\vec{r}, \vec{V}, t) d \vec{V},
\end{aligned}
$$




$$
q_{t r}=\int c^{2} \vec{c} f_{1}(\vec{r}, \vec{V}, t) d \vec{V}, \vec{q}_{\mathrm{rot}}=\int \vec{c} f_{2}(\vec{r}, \vec{V}, t) d \vec{V}, \vec{q}_{v i b}=\int \vec{c} f_{3}(\vec{r}, \vec{V}, t) d \vec{V}
$$

Here, $m$ denotes the molecular mass, $R$ is the gas constant, $\vec{c}$ denotes the molecular thermal velocity, $k$ is the Boltzmann constant, $\delta_{i j}$ is the Kronecker symbols, $i, j=1,2,3$.

All flow variables are evaluated and updated by the three reduced non-equilibrium VDFs of $f_{1}, f_{2}$ and $f_{3}$ over the velocity space, and can be non-dimensionalized, in which the characteristic variables are referred to its free-stream equilibrium values at infinity, such as number density $n_{\infty}$, temperature $T_{\infty}$, the most probable velocity $c_{m \infty}=\sqrt{2 R T_{\infty}}$, time $t_{\infty}=L / c_{m \infty}$, where $L$ is a characteristic length of the problem, force $m n_{\infty} c_{m \infty}^{2} / 2$, heat flux $m n_{\infty} c_{m \infty}^{3} / 2$ and the distribution function $n_{\infty} / c_{m \infty}^{3}$. And the Knudsen number is defined as $K n=\lambda_{\infty} / L$, where $\lambda_{\infty}$ is the mean free path in the free stream flow.

The Boltzmann model Eq. (1) can be transferred into a group of partial differential equations with non-homogeneous and hyperbolic conservation [24, 35, 37-39] on time and physical space at every DVO points.

$$
\frac{\partial U}{\partial t}+\frac{\partial F}{\partial \xi}+\frac{\partial G}{\partial \eta}+\frac{\partial H}{\partial \varsigma}=S
$$

with,

$$
U=J \cdot\left[\begin{array}{l}
f_{1, \sigma, \delta, \theta}(t, x, y, z) \\
f_{2, \sigma, \delta, \theta}(t, x, y, z) \\
f_{3, \sigma, \delta, \theta}(t, x, y, z)
\end{array}\right], F=\bar{U} U, G=\bar{V} U, H=\bar{W} U
$$

Where,

$$
\begin{aligned}
\bar{U} & =V_{x \sigma} \xi_{x}+V_{y \delta} \xi_{y}+V_{z \theta} \xi_{z}, \bar{V}=V_{x \sigma} \eta_{x}+V_{y \delta} \eta_{y}+V_{z \theta} \eta_{z}, \bar{W}=V_{x \sigma} \zeta_{x}+V_{y \delta} \zeta_{y}+V_{z \theta} \zeta_{z}, \\
J & =\partial(x, y, z) / \partial(\xi, \eta, \zeta) .
\end{aligned}
$$

Under the frame of the GKUA, the LU-SGS implicit schemes based on the cell central-type finite volume method (FVM) [6] are constructed to directly solve the Boltzmann model Eq. (11). Using the FVM, in the cell central-type control volume $\Omega_{I J}$, the integral equation can be got for the exemplification of $2 \mathrm{D}$ flows,

$$
\frac{\partial \bar{f}_{I J}}{\partial t}+\frac{1}{\Omega_{I J}} \oint_{\partial \Omega} \vec{F} \cdot \vec{n} d s=\bar{S}_{I J}
$$

Here, $\vec{F}=\left(V_{x \sigma} f_{\sigma, \delta}\right) \vec{i}+\left(V_{y \delta} f_{\sigma, \delta}\right) \vec{j}, \vec{n}$ is the normal vector on boundaries of the control volume, $\bar{X}_{I J}$ is the average value of $X_{I J}$ on $\Omega_{I J}$.

The second term in the left hand side of Eq. (12) can be rewritten as: 


$$
\begin{gathered}
\frac{1}{\Omega_{I J}} \oint_{\partial \Omega} \vec{F} \cdot \vec{n} d s=\frac{1}{\Omega_{I J}}\left(H_{I+1 / 2, J}-H_{I-1 / 2, J}+H_{I, J+1 / 2}-H_{I, J-1 / 2}\right) \\
H_{I+1 / 2, J}=\left[n_{x} V_{x} f+n_{y} V_{y} f\right]_{I+1 / 2, J} \Delta s_{I+1 / 2, J}=A_{I+1 / 2, J} f_{I+1 / 2, J} \\
A_{I+1 / 2, J}=\left[n_{x} V_{x}+n_{y} V_{y}\right]_{I+1 / 2, J} \Delta s_{I+1 / 2, J}
\end{gathered}
$$

After obtaining $f_{I J}^{n+1}$ in every control volume, all of the macroscopic flow variables can be evaluated and updated by the quadrature methods [35-38], such as the Gauss-Legendre numerical quadrature method. Then, the integration of the distribution function over the discrete velocity domain $\left[V_{a}, V_{b}\right]$ can be solved by the following extended Gauss-Legendre formula,

$$
\begin{aligned}
& \int_{V_{a}}^{V_{b}} f(V) d V=\sum_{k=1}^{N} \int_{V_{k}}^{V_{k+1}} f(V) d V, \\
& \int_{V_{k}}^{V_{k+1}} f(V) d V \approx \frac{V_{k+1}-V_{k}}{2} \sum_{i=1}^{n} A_{i} f\left(\frac{V_{k+1}+V_{k}}{2}+\frac{V_{k+1}-V_{k}}{2} x_{i}\right) .
\end{aligned}
$$

The time step $\Delta t$ in the implicit scheme is less strict than that of the explicit schemes. In this study, the time step is set as

$$
\Delta t=C F L \cdot \min _{i}\left(l_{i}\right) / \max _{\sigma, \delta}\left(V_{x \sigma}, V_{y \delta}\right)
$$

Where, the CFL number is set to $0.95 . l_{i}$ is the characteristic linear size of the $i$-th grid element. The $V_{x \sigma}$ and $V_{y \delta}$ are the discretized velocity components in $V_{x^{-}}$and $V_{y^{-}}$ directions, respectively.

To resolve the difficulty of the vast computer memory required by the current algorithm in solving three-dimensional complex flows and to well exploit massive power of parallel computers, the multi-processing strategy and parallel implementation technique suitable for the GKUA are investigated by using the technique of domain decomposition. For the velocity domain $\Omega_{V}$ decomposition strategy, the amount of computation for the discrete velocity quadrature integrations for macroscopic quantities occupies about only one fifth of the total amount of the computation of the whole GKUA. Accordingly, the number of CPU processors can reach the number of DVO points of $N_{\sigma} \times N_{\theta} \times N_{\delta}$, then one can realize super-parallel computing. Thus, it is suggested that the velocity space $\Omega_{V}$ decomposition technique to be adopted. The numerical experience indicates that in each CPU the amount of work and the memory required for handling the solution of the distribution function is about $1 / N_{p}$ of serial computing, where the $N_{p}$ is the number of processors participated in the parallel computing. This overcomes the restriction of memory problem in serial computation and is of crucial importance in computing complex 3-D hypersonic rarefied flows. When the number of DVO points used is set and the way for the parallel distribution of discrete velocity space is generated, each processor will have its correspondingly individual integrality in solving the discrete VDFs assigned to it and the degree of parallelization is higher. This belongs to a coarse-grain high performance parallel computing scheme. 
To illustrate and test the feasibility and parallel efficiency of large scale 3-D flow computation based on the present GKUA for solving the unified Boltzmann model equation, here we report our experience of such calculation on the Sunway BlueLight MPP parallel supercomputer with up to 8704 ShenWei SW1600 processors using the parallel strategies described above. The discrete velocity space MPI parallelism is designed and combined with core-level Open ACC paralleling on dimension of location space. Recently, the hardware parallel speedup technique has been applied to accelerate computation of the GKUA. Figure 2a shows the speedup ratio of parallel calculation with numbers of CPU of (a). n=1440 7920CPU and (b). 4950 20625CPU, where the dashed line indicates the ideal speedup ratio and the solid line depicts the present one. A more recent large-scale computation of the complex hypersonic flow over the Tiangong-1 two-capsule vehicle based on the GKUA with six-dimensional phase space grid system of $101 \times 61 \times 31 \times 120 \times 90 \times 70$ is put in practice with $500 \sim 45,000$ CPUs. The speed-up ratio and corresponding parallel efficiency are shown in Fig. 3. It can be shown from Figs. 2 and 3 that the parallel speed-up almost goes up as near-linearity with the increase of the number of CPU. This indicates that the parallel computation of the GKUA possesses very high parallel efficiency and scalability with good load balance and data communication efficiency by the use of the present parallel computing scheme. From these tests on small, moderate and large scale parallel computing facilities for the domain decomposition strategy of the GKUA, the measured speedup ratio is very close to the theoretical one, and at least $80 \%$ parallel efficiency can be achieved for all various scale computations. This validates the present GKUA methods possess quite high parallel speedup and parallel efficiency. Good parallel speedup performance can ensure to enlarge the computational job scale by increasing the number of processors with high parallel efficiency and expandability. This enables us to perform extensive and large scale 3-D hypersonic flow simulations for the uncontrolled Tiangong-1 spacecraft at various flight trajectory points, although the computation is expensive and almost all the largest computing facilities in the High-Performance Supercomputer Centers in China are utilized.

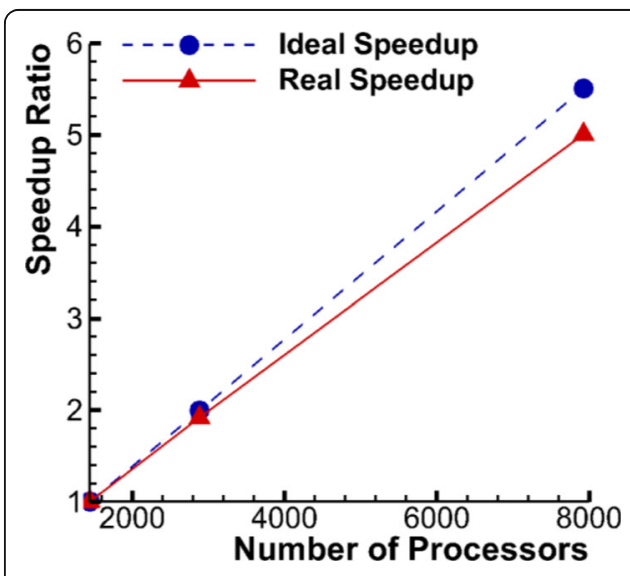

(a) $1440 \sim 7920 \mathrm{CPU}$

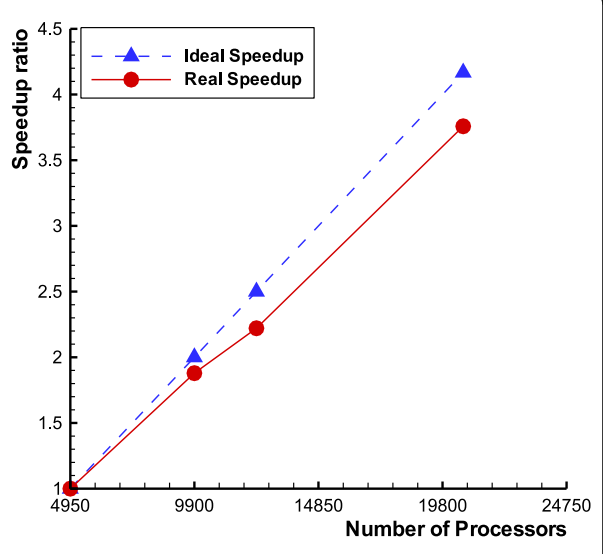

(b) $4950 \sim 20625 \mathrm{CPU}$

Fig. 2 Speedup ratio of parallel computation for GKUA with 1440 7920 20625 processors. a 1440 7920CPU, b 4950 20625CPU 


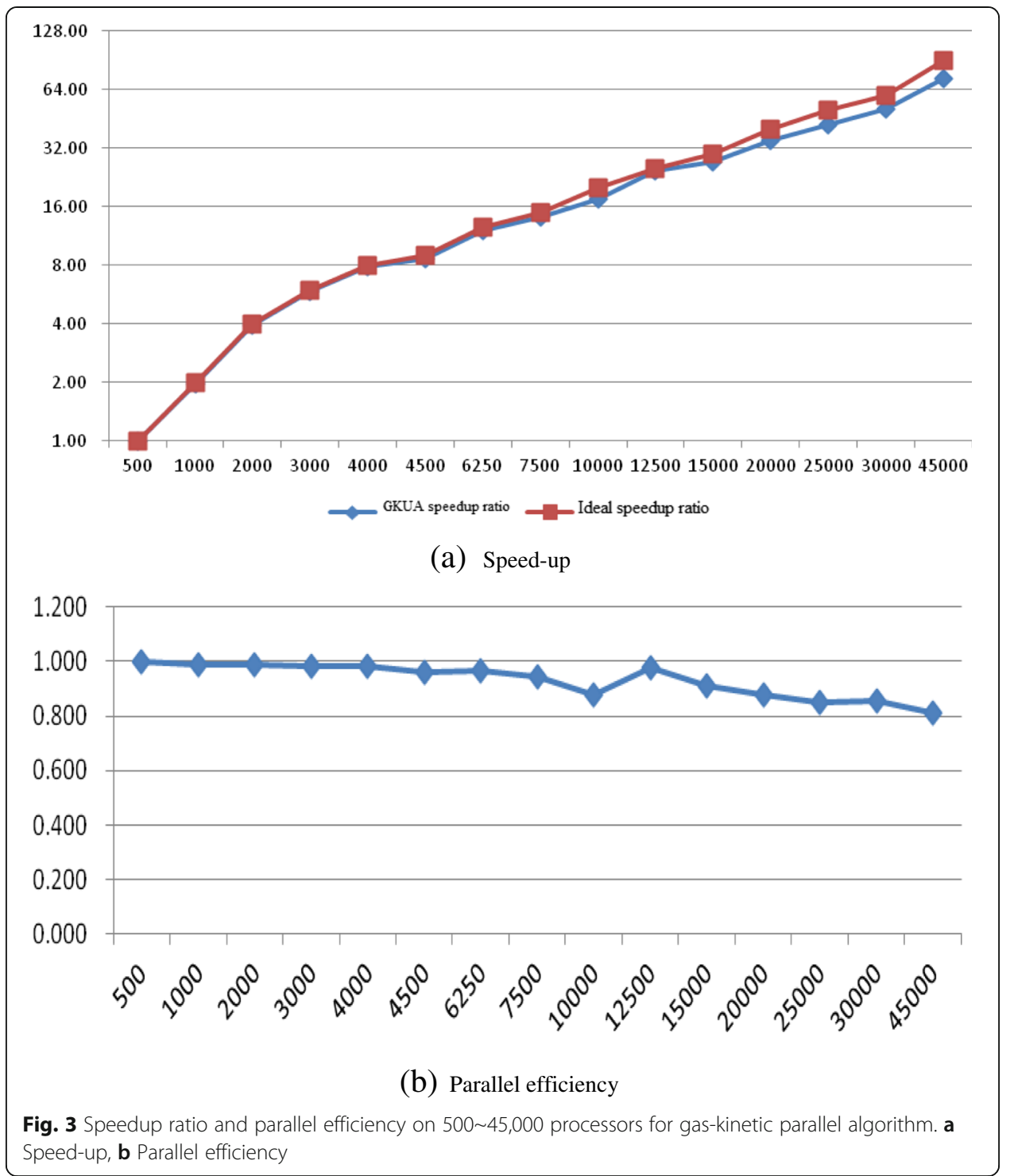

\section{Finite element algorithm for structural deformation and thermal damage under hypersonic aerothermodynamic environment}

Once the hypersonic aerothermodynamic environment is obtained around the large-scale reentering spacecraft, the finite-element methods to simulate the heat conduction and the dynamic deformation and destructive behavior of the metal truss structure need to be developed. In the three-dimensional domain $\Omega \subset \mathbb{R}^{3}$, the dynamic thermoelasticity equation $[41,42]$ is expressed, as follows

$$
\rho \frac{\partial^{2} u_{i}}{\partial t^{2}}+\tau \frac{\partial u_{i}}{\partial t}-\frac{\partial}{\partial x_{j}}\left(C_{i j k l} \frac{\partial u_{k}}{\partial x_{l}}\right)+\frac{\partial}{\partial x_{j}}\left(\beta_{i j} \theta\right)=f_{i}
$$

Where, $\boldsymbol{u}=\left[\begin{array}{lll}u_{1} & u_{2} & u_{3}\end{array}\right]^{T}$ is the displacement vector, $\boldsymbol{f}=\left[\begin{array}{lll}f_{1} & f_{2} & f_{3}\end{array}\right]^{T}$ is the volume force and the superscript ${ }^{T}$ denotes transposition. $\rho$ is the density, $\tau$ is the damping coefficient and $C_{i j k l}$ is the 4-order elasticity tensor. For homogeneous and isotropic materials, $C_{i j k l}$ is written as $C_{i j k l}=\lambda \delta_{i j} \delta_{k l}+G\left(\delta_{i k} \delta_{j l}+\delta_{i l} \delta_{j k}\right)$. Here, $\delta_{i j}$ is the Kronecker 
symbol, $\lambda$ and $G$ are the Lamé constants, expressed by the Young's modulus $E$ and the Possion's ration $v$ as.

$$
\lambda=\frac{E v}{(1+v)(1-2 v)}, G=\frac{E}{2(1+v)} .
$$

$\theta=T-T_{0}$ is the temperature increment with $T$ and $T_{0}$ denoting the absolute temperature and reference temperature respectively and $\beta_{i j}$ is the thermo-stress module, $\beta_{i j}=C_{i j k l} \alpha_{k l}$, where $\alpha_{k l}$ is the thermal expansion coefficient. For homogeneous and isotropic materials, $\alpha_{k l}=\alpha_{0} \delta_{k l}, \alpha_{0}$ is the thermal expansion constant, and then $\beta_{i j}$ can be written as $\beta_{i j}=3 \gamma \alpha_{0} \delta_{i j}$, here $\gamma=\frac{E}{3(1-2 v)}$ is the bulk modulus, which means that the increment or decrement of the temperature leads to the expansion or contraction of the body, effecting the stress distribution of the structure. The elastic strain $\varepsilon_{i j}$ is

$$
\varepsilon_{i j}=\frac{1}{2}\left(\frac{\partial u_{i}}{\partial x_{j}}+\frac{\partial u_{j}}{\partial x_{i}}\right)
$$

and the stress tensor $\sigma_{i j}=C_{i j k l} \varepsilon_{k l}-\beta_{i j} \theta$.

Considering the deforming influence on the temperature field, the transient heat conductive equation is expressed as follows

$$
\rho c \frac{\partial \theta}{\partial t}+T_{0} \beta_{i j} \frac{\partial}{\partial t}\left(\frac{\partial u_{i}}{\partial x_{j}}\right)-\frac{\partial}{\partial x_{j}}\left(k_{i j} \frac{\partial \theta}{\partial x_{i}}\right)=h .
$$

Here, $c$ is the specific heat, $k_{i j}$ denotes the 2-order conductivity tensor and $h$ is the heat source. Considering also homogeneous and isotropic materials, from the expression of $\beta_{i j}$, the second term of Eq. (20) can be denoted by

$$
T_{0} \beta_{i j} \frac{\partial}{\partial t}\left(\frac{\partial u_{i}}{\partial x_{j}}\right)=T_{0} \frac{E \alpha_{0}}{1-2 v} \frac{\partial \varepsilon_{i i}}{\partial t}
$$

in which $\varepsilon_{i i}$ is the volumetric strain, Eq. (21) illustrates that the change rate of the volumetric strain is the positive correlation with the temperature field.

In summary, for the convenience of our derivation, the weak form of the dynamic thermoelasticity coupling equations is to find $\boldsymbol{u}$ and $\theta$, such that

$$
\left\{\begin{array}{l}
\int_{\Omega} \rho \frac{\partial^{2} u_{i}}{\partial t^{2}} \tilde{u}_{i} d x+\int_{\Omega} \tau \frac{\partial u_{i}}{\partial t} \tilde{u}_{i} d x+\int_{\Omega} C_{i j k l} \frac{\partial u_{k}}{\partial x_{l}} \frac{\partial \tilde{u}_{i}}{\partial x_{j}} d x-\int_{\Omega} \beta_{i j} \theta \frac{\partial \tilde{u}_{i}}{\partial x_{j}} d x=\int_{\Omega} f_{i} \tilde{u}_{i} d x+\int_{\Gamma_{2}} p_{i} \tilde{u}_{i} d s \\
\int_{\Omega} \frac{\rho c}{T_{0}} \frac{\partial \theta}{\partial t} \tilde{\theta} d x+\int_{\Omega} \beta_{i j} \frac{\partial^{2} u_{i}}{\partial t \partial x_{j}} \tilde{\theta} d x+\int_{\Omega} \frac{k_{i j}}{T_{0}} \frac{\partial \theta}{\partial x_{j}} \frac{\partial \tilde{\theta}}{\partial x_{i}} d x=\int_{\Omega} \frac{h}{T_{0}} \tilde{\theta} d x-\int_{\Gamma_{2}^{\prime}} \frac{q}{T_{0}} \tilde{\theta} d s .
\end{array}\right.
$$

From the equations above, it is noted that the displacement $\boldsymbol{u}$ and temperature increment $\theta$ are dependent on each other, which have to be solved simultaneously in the finite-element method. Equation Section (Next)

$$
\left\{\begin{array}{l}
\boldsymbol{M}_{u} \ddot{\boldsymbol{d}}(t)+\boldsymbol{C}_{u} \dot{\boldsymbol{d}}(t)+\boldsymbol{K}_{u} \boldsymbol{d}(t)-\boldsymbol{L} \boldsymbol{\xi}(t)=\boldsymbol{F}(t) \\
\boldsymbol{M}_{\theta} \dot{\boldsymbol{\xi}}(t)+\boldsymbol{K}_{\theta} \boldsymbol{\xi}(t)+\boldsymbol{L}^{T} \dot{\boldsymbol{d}}(t)=\boldsymbol{G}(t)
\end{array}\right.
$$

Adopting the Newmark implicit method $[2,5]$ to deal with the velocity and acceleration terms, we have the time stepping scheme 


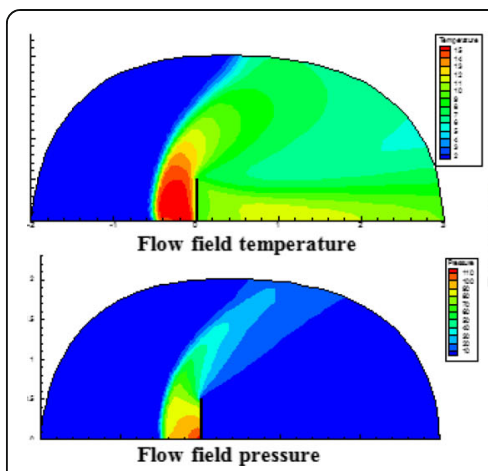

(a) Hypersonic flow field

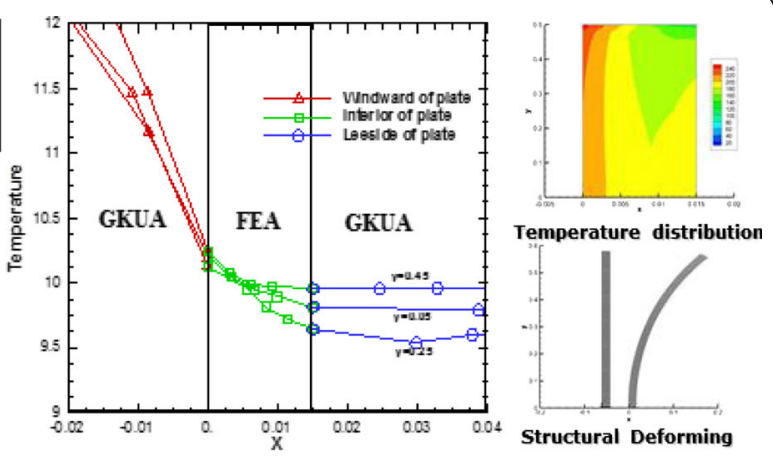

(b) Temperature correlation along with $\mathrm{X}$

(c) Structural deformation

Fig. 4 Temperature contours and correlation along with $X$ at different $y=0.06,0.26$ and 0.46 between flow field and inner structure. a Hypersonic flow field, b Temperature correlation along with X, c Structural deformation

$$
\begin{aligned}
\left(\frac{1}{\eta \Delta t^{2}} \boldsymbol{M}_{\boldsymbol{u}}+\frac{\omega}{\eta \Delta t} \boldsymbol{C}_{\boldsymbol{u}}+\boldsymbol{K}_{\boldsymbol{u}}\right) \boldsymbol{d}^{n+1}-\boldsymbol{L} \boldsymbol{\xi}^{n+1} & =\boldsymbol{F}^{n+1}+\boldsymbol{M}_{\boldsymbol{u}}\left(\frac{1}{\eta \Delta t^{2}} \boldsymbol{d}^{n}+\frac{1}{\eta \Delta t} \dot{\boldsymbol{d}}^{n}+\left(\frac{1}{2 \eta}-1\right) \ddot{\boldsymbol{d}}^{n}\right) \\
& +\boldsymbol{C}_{\boldsymbol{u}}\left(\frac{\omega}{\eta \Delta t} \boldsymbol{d}^{n}+\left(\frac{\omega}{\eta}-1\right) \dot{\boldsymbol{d}}^{n}+\left(\frac{\omega}{2 \eta}-1\right) \Delta t \ddot{\boldsymbol{d}}^{n}\right) .
\end{aligned}
$$

where the parameters $0 \leq \omega \leq 1,0 \leq \eta \leq 1 / 2$, and usually when $\omega \geq 0.5$ and $\eta \geq 0.25(0.5$ $+\omega)^{2}$, the scheme above is unconditionally stable.

To ensure that the displacement and the temperature distribution of the inner structure of hypersonic flying body are continuously solved from Eq. (24), the unified simulation of structural deformation and hypersonic aerothermodynamics needs to be constructed with real-time trajectory updating, in which the values of the aerothermodynamic temperature and force computed by the GKUA as the boundary conditions for the coupled thermoelasticity problems. To combine the present FEA methods with the GKUA [6, 35, 37-39, 45] of aerothermodynamics covering various flow regimes for simplicity, we consider and verify the flow-rounded infinite plate problem as an example of thermodynamic coupling response during spacecraft re-entering Earth's atmosphere, shown in Fig. 4. The size of the plate is $0.015 \mathrm{~m} \times 0.5 \mathrm{~m}$, the exterior of which is the reentry near-continuum transition flow, the inflowing Mach number, Knudsen number and ratio of specific heat are $M a_{\infty}=8.3666$, $K n_{\infty}=0.01$ and $\gamma=1.4$, respectively. The exterior flow field of the plate with the mesh of $63 \times 41$ in the $\mathrm{XoY}$ coordinate is computed by the GKUA for solving the unified Boltzmann model equation. Because of the re-entry near-continuum transition flow regime, the thick detached shock wave layer is formed around the thin plate shown in Fig. 4a, and the strong aerodynamic heating and force are imposed as the interface condition of the present thermodynamic coupling finite-element algorithm on the plate surface. The high temperature and strong pressure make the plate expand, deform and damage.

\section{Results and discussion}

To verify the accuracy and reliability of the present computable modeling of Boltzmann equation and GKUA in solving thermodynamic non-equilibrium flows with vibrational energy excitation, the cylinder flows of Nitrogen gas with $K n_{\infty}=0.01, M a_{\infty}=5, n_{\infty}=$ $1.4966 E 20 / \mathrm{m}^{3}, T_{\infty}=T_{w}=500 \mathrm{k}$ are solved by the present GKUA and the DSMC, 


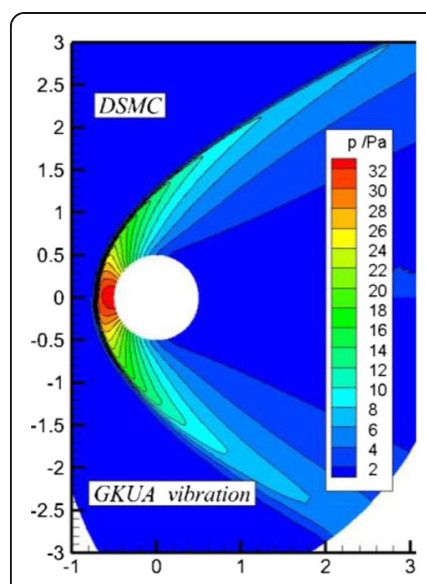

(a) Pressure

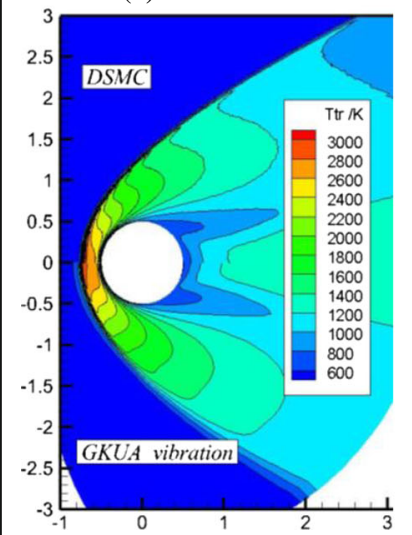

(d) Translational Temp.

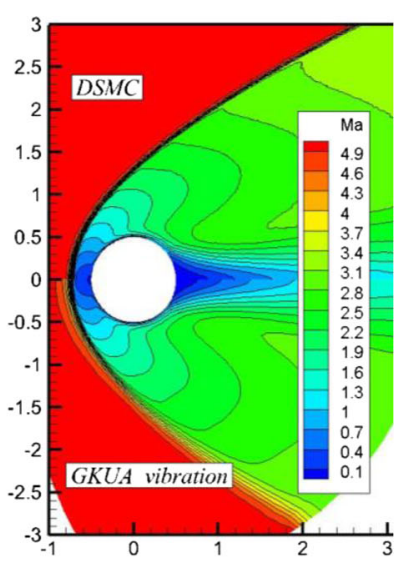

(b) Mach number

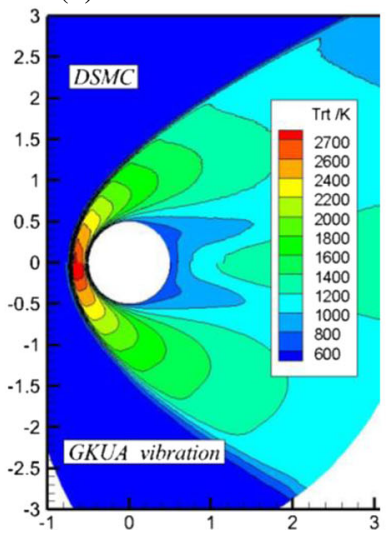

(e) Rotational Temp.

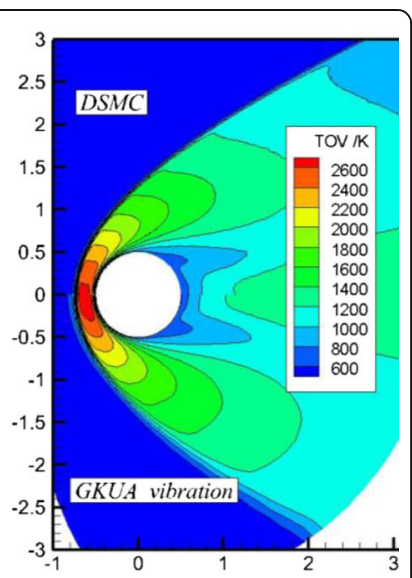

(c) Overall Temp.

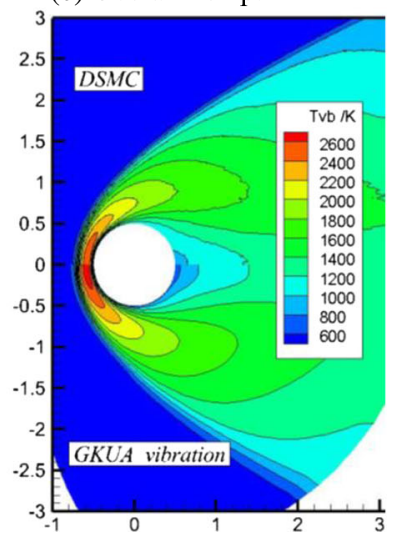

(f) Vibrational Temp.

Fig. 5 Macro-variables for cylinder flows of $K n_{\infty}=0.01, M a_{\infty}=5$ solved by GKUA comparing with DSMC. a Pressure, b Mach No., c Overall Temp., d Translational Temp., e Rotational Temp., f Vibrational Temp

respectively. Figure 5 shows macroscopic flow variables including (a) pressure, (b) Mach number, (c) overall temperature, (d) translational temperature, (e) rotational temperature and (f) vibrational temperature with the comparison of the DSMC results, in which the two results agree well, especially, the contours of pressure and overall temperature distribution are almost completely identical. It is indicated from the translational, rotational, vibrational and overall temperature distribution that the translational temperature is the highest, the rotational temperature is the second and the vibrational temperature is the lowest in the interior stagnation region after the detached shock wave, and the vibrational temperature computed by the present GKUA with better resolution is slightly higher than the DSMC results.

In addition, the present GKUA uses the structured mesh with refinement near surface and slightly wider grid in the location of detached shock wave, however, the DSMC uses self-adaptive unstructured mesh and the space mesh is refined to better capture shock wave where the macroscopic flow gradient is large, so that there is a slight difference in the shock location between the two methods. Figure 6 shows the surface pressure and heat flux distribution with different non-equilibrium effects including the GKUA results with the Shakhov model of simple gas, the ES model of rotational excitation polyatomic gas and Nitrogen vibrational energy excitation, respectively, where the "DSMC-translation", 


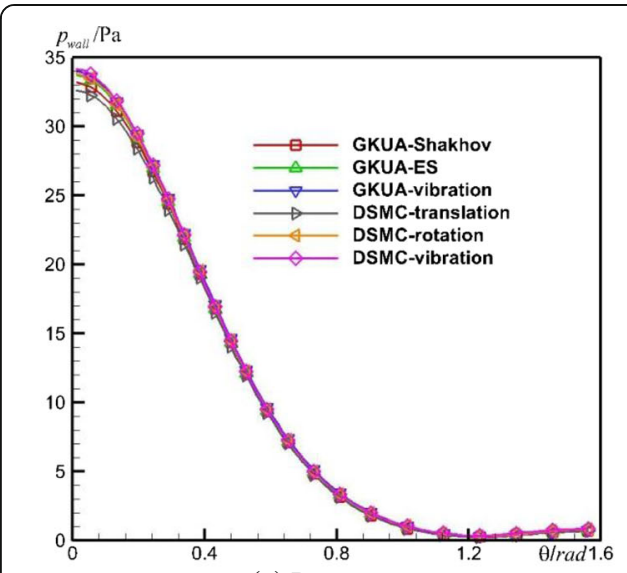

(a) Pressure

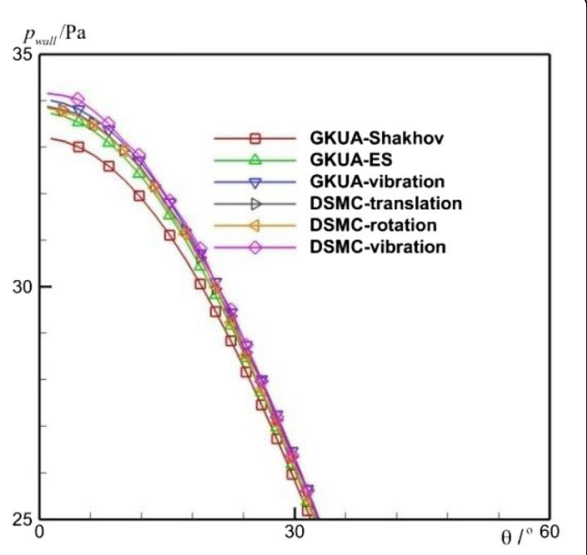

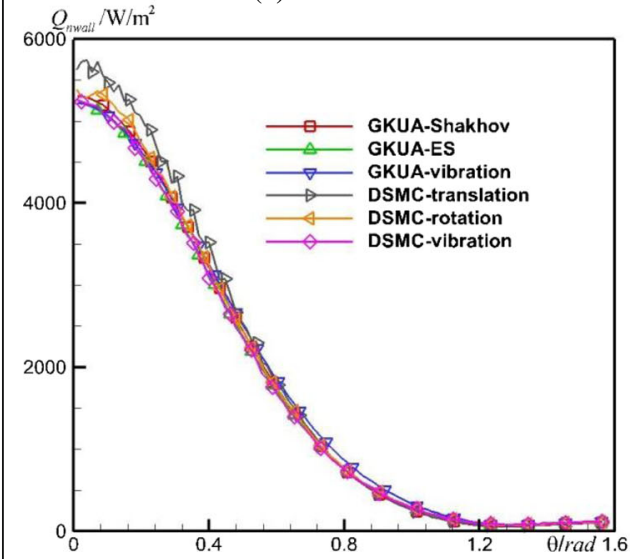

(c) Heat flux (b) Enlarged near stagnation point for pressure

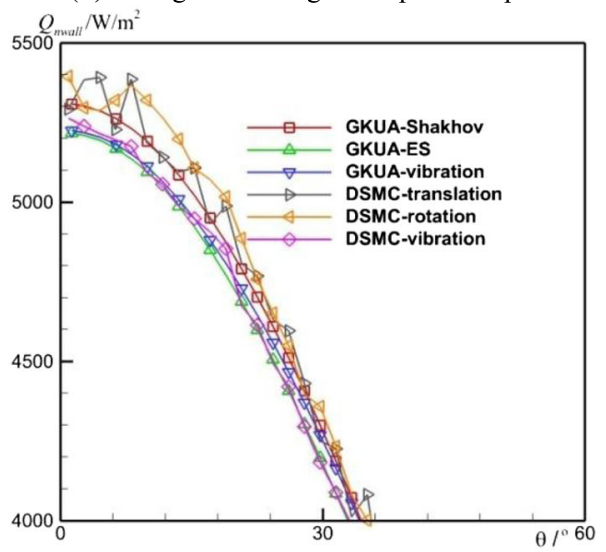

(d) Enlarged near stagnation point for heat flux

Fig. 6 Surface pressure and heat flux for cylinder flows of $K n_{\infty}=0.01, M a_{\infty}=5$ solved by GKUA comparing with DSMC results with different non-equilibrium effects with the Shakhov model of simple gas, the ES model of rotational excitation polyatomic gas and the Nitrogen vibrational energy excitation, respectively. a Pressure, b Enlarged near stagnation point for pressure, $\mathbf{c}$ Heat flux, d Enlarged near stagnation point for heat flux

"DSMC-rotation" and "DSMC-vibration" denote the DSMC results of the Nitrogen as a simple gas without excitation of internal energy, the Nitrogen gas with rotational energy and the Nitrogen gas with vibrational energy excitation, respectively, and Fig. $6 \mathrm{~b}$ and d correspond to the local enlarged profiles of surface pressure and heat flux near the stagnation point. It is indicated that the surface pressure distribution near the stagnation point is the lowest for the "DSMC-translation", the next for the "GKUA-Shakhov", and other results almost coincide. The surface heat flux distribution shown in Fig. $6 \mathrm{c}$ and $\mathrm{d}$ for the "DSMC-translation" and "DSMC-rotation" near the stagnation point is obviously higher than the other results and exists serious statistical fluctuation. On the whole, the results obtained by these methods are in very close agreement.

In order to study the influence of vibration energy excitation on the aerodynamics of Tiangong-type spacecraft with solar array, Fig. 7 shows (a) translational temperature, (b) rotational temperature, (c) vibrational temperature, (d) Overall Temp. (e) Mach No. and (f) streamline structure around the Tiangong-type spacecraft with $\mathrm{H}=90 \mathrm{~km}, M a_{\infty}$ $=8$ in thermodynamic non-equilibrium effect of vibrational energy excitation solved by the present GKUA in massively parallel computing. It is indicated that the vortex structure is formed between the solar array and the module, and the axial force and normal 


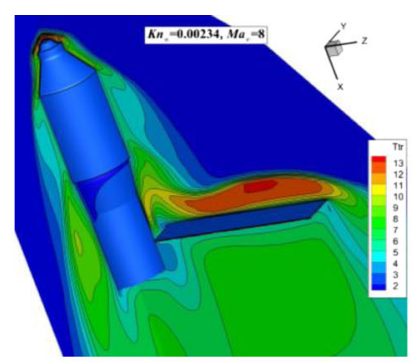

(a) Translational Temp.

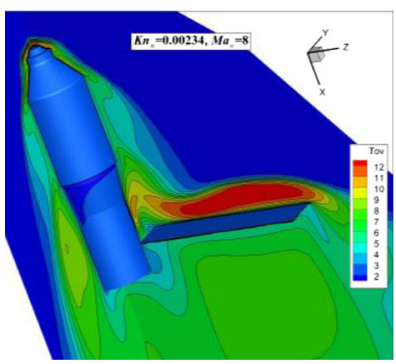

(d) Overall Temp.

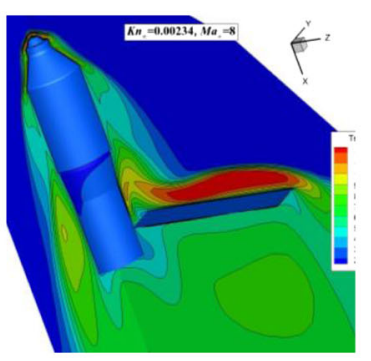

(b) Rotational Temp.

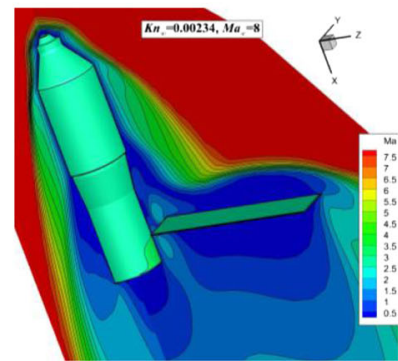

(e) Mach No.

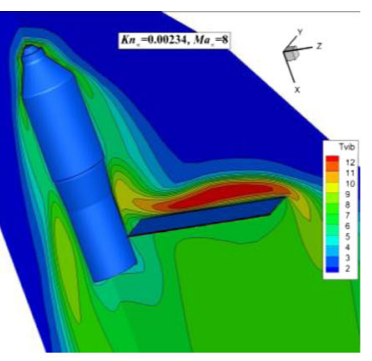

(c) Vibrational Temp.

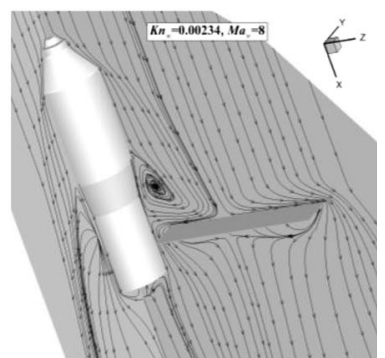

(f) Streamline structure

Fig. 7 Macro-variables around Tiangong-type spacecraft with $\mathrm{H}=90 \mathrm{~km}, M a_{\infty}=8$ with thermodynamic nonequilibrium. a Translational Temp., b Rotational Temp., c Vibrational Temp., d Overall Temp., e Mach No., f Streamline structure

force coefficients are respectively obtained as $\mathrm{Ca}=9.635$ and $\mathrm{Cn}=-1.525$ for thermodynamic non-equilibrium gas, and $\mathrm{Ca}=9.535$ and $\mathrm{Cn}=-1.795$ for completely simple gas with the relative error of $1.04 \%$ and $17.7 \%$, in which the complex non-equilibrium flow between the solar panels and the modules has a great influence on the normal force around the Tiangong-type spacecraft.
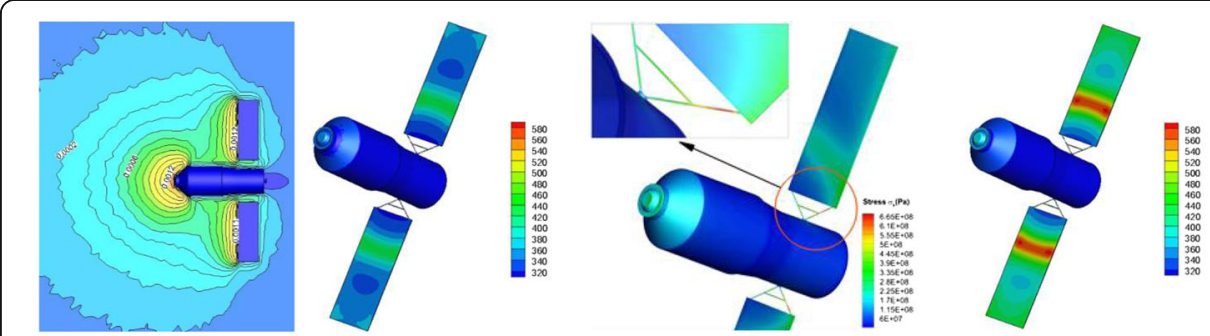

(a)Pressure distr. of $\mathrm{H}=250 \mathrm{~km}$ (b)Struct. Temp. of $\mathrm{H}=120 \mathrm{~km}$ (c) Normal stress (d) Struct. Temp. of $\mathrm{H}=110 \mathrm{~km}$

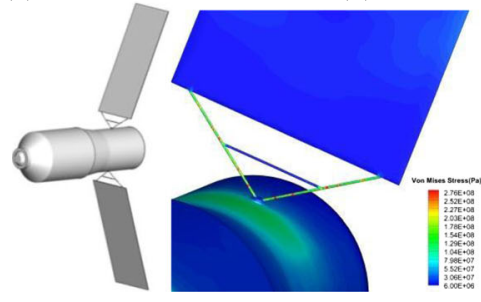

(e) Structural deformation of $\mathrm{H}=100 \mathrm{~km}$

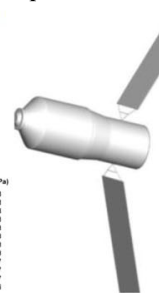

(f) Deformation of $\mathrm{H}=95 \mathrm{~km}$

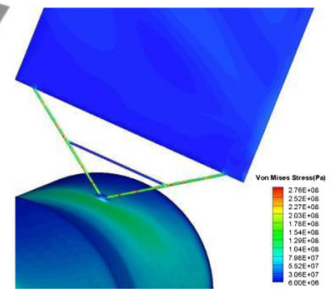

(g) Structural Temp. of $\mathrm{H}=90 \mathrm{~km}$

Fig. 8 Pressure contours of flow field, temperature distribution and deformation of structure during the first disintegration of $\mathrm{H}=250 \sim 90 \mathrm{~km}$ around Tiangong-1 spacecraft. a Pressure distr. of $\mathrm{H}=250 \mathrm{~km}, \mathbf{b}$ Struct. Temp. of $\mathrm{H}=120 \mathrm{~km}$, $\mathbf{c}$ Normal stress, $\mathbf{d}$ Struct. Temp. of $\mathrm{H}=110 \mathrm{~km}$, e Structural deformation of $\mathrm{H}=100$ $\mathrm{km}, \mathbf{f}$ Deformation of $\mathrm{H}=95 \mathrm{~km}, \mathbf{g}$ StructuralTemp. of $\mathrm{H}=90 \mathrm{~km}$ 


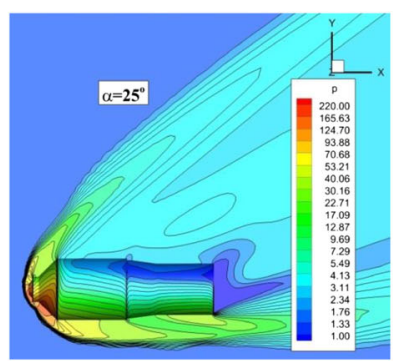

(a) Pressure

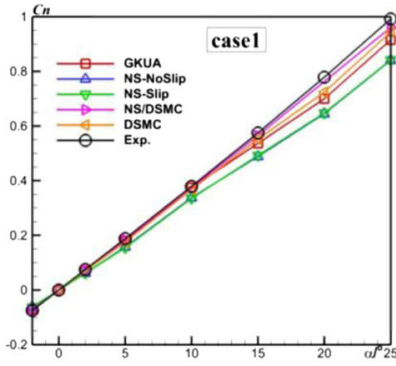

(d) Normal force coefs

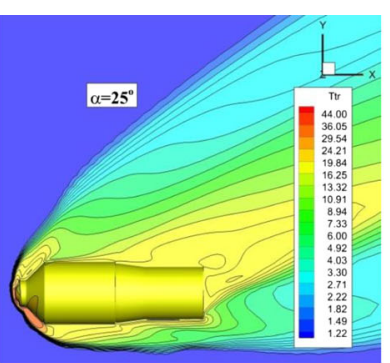

(b) Temperature

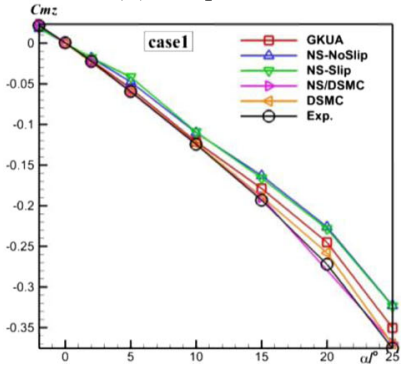

(e) Pitching moment coefs.

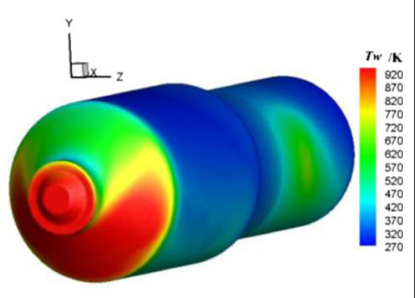

(c) Structural Temp. at $801 \mathrm{~s}$

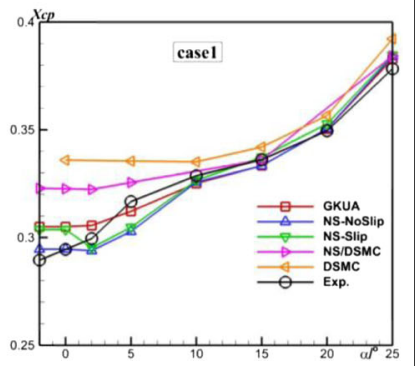

(f) Pressure center position

Fig. 9 Validation of computation and experiment for aerodynamics on the two-capsule vehicle of Tiangong spacecraft $\left(H=62.1 \mathrm{~km}, M a_{\infty}=12.79, K n_{\infty}=3.37 \times 10^{-5}\right)$. a Pressure, b Temperature, $\mathbf{c}$ Structural Temp. at $801 \mathrm{~s}$, d Normal force coefs, e Pitching moment coefs, f Pressure center position

To simulate metal-truss structure deformation and damage under the strong aerothermodynamic environment during the reentry falling process of the large-scale low-orbit vehicle with solar array from $\mathrm{H}=250 \mathrm{~km}$, Fig. 8 shows the pressure contours around Tiangong-type spacecraft with $K n_{\infty}=220.34, M a_{\infty}=22.75$, and the temperature, normal stress and deformation distribution of Tiangong-type spacecraft structure varied with falling height from $\mathrm{H}=120 \mathrm{~km}$ to $\mathrm{H}=90 \mathrm{~km}$ during the first disintegration.

To compare and verify the correctness of different algorithm models and experiments in solving the common hypersonic flows around the two-capsule body of Tiangong-type spacecraft after disintegration for the first time, Fig. 9a, b and c show the pressure and temperature contours of flow field and the structural temperature of two-capsule vehicle of Tiangong- 1 spacecraft computed by the GKUA at $801 \mathrm{~s}$ during the reentry, and Fig. $9 \mathrm{~d}-\mathrm{f}$ show the aerodynamics solved by the GKUA, DSMC [9], N-S/DSMC [43], Slip N-S and low-density wind-tunnel experiments [44] for hypersonic flows around the two-capsule of Tiangong-type spacecraft, in which good agreement exists and the GKUA results are more identical to the experimental data and the DSMC results, in which the GKUA has been
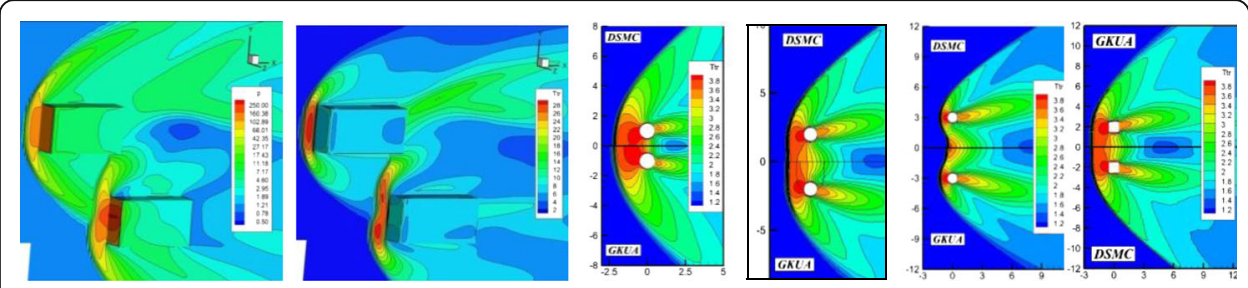

Fig. 10 Macro flow variables around component and debris from multiple disintegration of Tiangong-1 


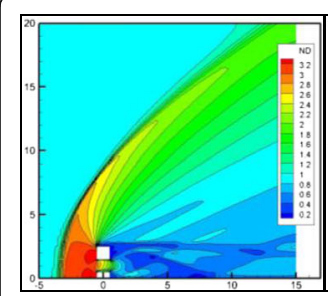

(a) Number density

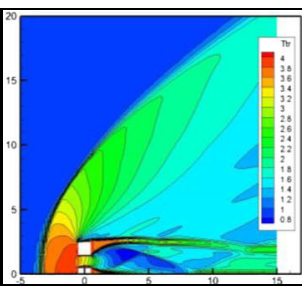

(b) Temperature

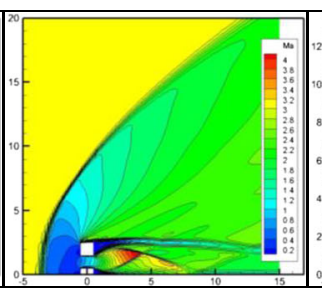

(c) Mach number

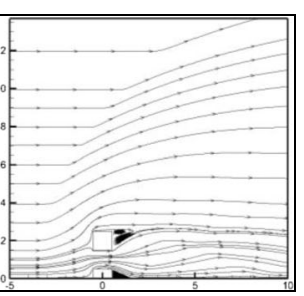

(d) Flow streamlines

Fig. 11 Contours of number density, temperature, Mach number and streamlines past three side-by-side square cylinder for $K n_{\infty}=0.001, M a_{\infty}=3$ with spacing $H=2 L$. a Number density, b Temperature, $\mathbf{c}$ Mach number, d Flow streamlines

verified in high-precision and strong simulation ability in solving the aerodynamics of irregular large-scale spacecraft covering various flow regimes.

Large-scale spacecraft such as the Tiangong-1 in uncontrolled falling will disintegrate into multi-bodies in near-space flying surrounding during its reentry passing through the atmosphere. By developing multi-block patched grid generation technique, a computational platform based on the GKUA with implicit scheme has been established to solve the multi-body flow problems covering various flow regimes. Figure 10 shows the multi-body interference and flow phenomena around different multi-bodies of the irregular disintegration/debris simulated by the GKUA. When $\mathrm{H}=6 \mathrm{~L}$, interference become very weak. When $\mathrm{H}=10 \mathrm{~L}$, the Mach stem disappears, and the two oblique shock waves are formed by head shock waves, the separation vortex disappear.

In order to establish more engineering processing approaches for the flow interference among disintegrated multi-bodies, three side-by-side square cylinder flows are simulated by the present GKUA for $K n_{\infty}=0.001, M a_{\infty}=3$ with zero attack angle. As previous, $\mathrm{H}$ is defined as the distance between the centers of the upper and middle square debris, and $\mathrm{L}$ is the square cylinder side length. The flow state cases with different gap spacing of $\mathrm{H}=2 \mathrm{~L}, 4 \mathrm{~L}, 6 \mathrm{~L}, 8 \mathrm{~L}$ and $10 \mathrm{~L}$ are simulated, respectively. Figures 11 , 12,13 and 14 show the number density, temperature, Mach number contours and the corresponding flow streamlines relative to the four cases of $\mathrm{H}=2 \mathrm{~L}, 6 \mathrm{~L}, 8 \mathrm{~L}$ and $10 \mathrm{~L}$, respectively. It can be seen that similar flow phenomena to the two side-by-side square cylinder flows are revealed in Figs. 11 and 12 for $\mathrm{H}=2 \mathrm{~L}$ and $6 \mathrm{~L}$, respectively. In addition, when $H=2 L$, a series of interference shock waves appear in the zone after the throat, disturb the tail flow, and lead to a very complex flow field. As $\mathrm{H}$ increases, the interference shock waves move backward, when $H=6 L$, the flow interference

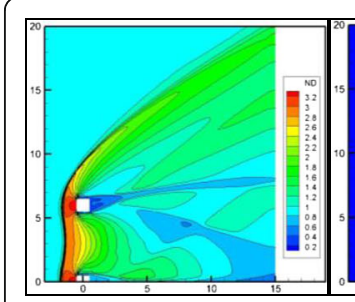

(a) Number density

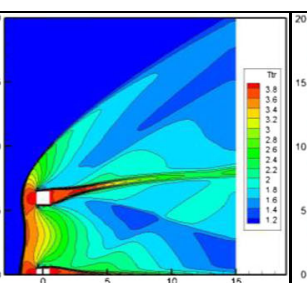

(b) Temperature

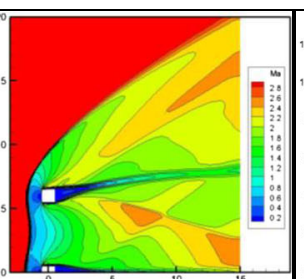

(c) Mach number

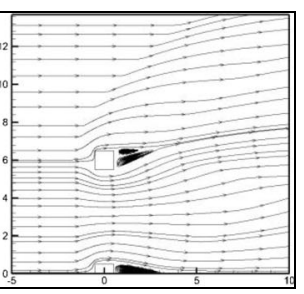

(d) Flow streamlines

Fig. 12 Contours of number density, temperature, Mach number and streamlines past three side-by-side square cylinder for $K n_{\infty}=0.001, M a_{\infty}=3$ with spacing $H=6 L$. a Number density, b Temperature, c Mach number, d Flow streamlines 


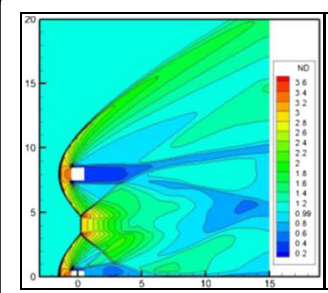

(a) Number density

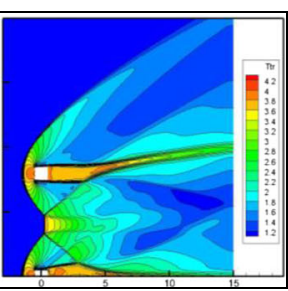

(b) Temperature

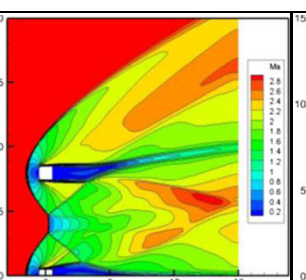

(c) Mach number

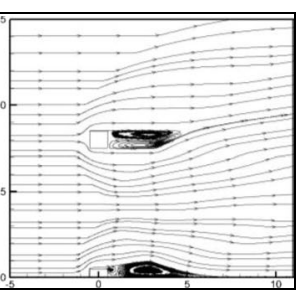

(d) Flow streamlines

Fig. 13 Contours of number density, temperature, Mach number and streamlines past three side-by-side square cylinder for $K n_{\infty}=0.001, M a_{\infty}=3$ with spacing $H=8 \mathrm{~L}$. a Number density, b Temperature, $\mathbf{c}$ Mach number, d Flow streamlines

interaction become very weak with saddle-type bow wave. When $\mathrm{H}$ increases to 8 $L$, a Mach stem wave system is formed by the interaction of the bow head shock waves. Behind the Mach stem, two oblique shock waves are formed and act on the tail flow, resulting in a series of shock waves and rarefaction waves. When $H=10 \mathrm{~L}$, the Mach stem disappears, and two oblique shock waves are formed by the detached head shock waves. It is indicated from the flow streamline structures of Figs. 11d, 12d and 13d that trailing vortices appear behind each square cylinder with the increase of gap spacing distance from $\mathrm{H}=2 \mathrm{~L}$ to $\mathrm{H}=8 \mathrm{~L}$, respectively, and because of flow interference among three side-by-side cylinders, the trailing vortices after the upper square cylinder are not symmetrical. The trailing vortices become large by increasing $\mathrm{H}$. When $H$ increases to $10 \mathrm{~L}$ (Fig. 14d), because of the shock interaction, a new separation vortex generates and appears at $x=7$ behind the middle square cylinder. At this time, the flow interference between the side-by-side square cylinders becomes very small, and the trailing vortexes behind the upper square cylinder are almost symmetrical. The separated vortex would disappear when $\mathrm{H}$ increases to a certain value. Figures 11, 12, 13 and 14 reveal the varying features of multi-body flow in the continuum flow regime with different gap spacing and the influential range of the flow interference among the multi-body side-by-side square debris.

Combined by developing the DSMC for hypersonic reentry thermochemical non-equilibrium flow, the N-S/DSMC, the slip N-S, and the computational methods of thermal environment, structural heat transfer/composite material pyrolysis in embedded layer or special device, and disassembly and separation with multi-body flow interference, the forecasting software for large-scale spacecraft

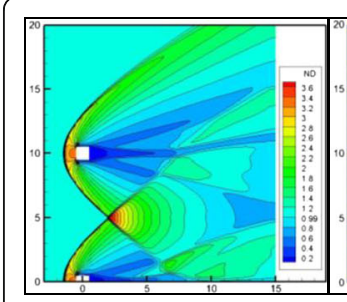

(a) Number density

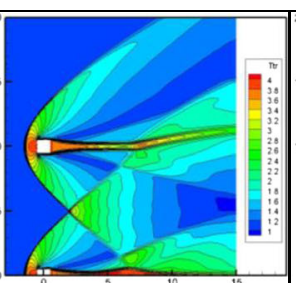

(b) Temperature

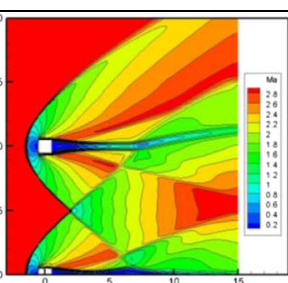

(c) Mach number

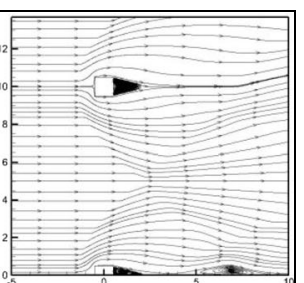

(d) Flow streamlines

Fig. 14 Contours of number density, temperature, Mach number and streamlines past three side-by-side square cylinder for $K n_{\infty}=0.001, M a_{\infty}=3$ with spacing $H=10 \mathrm{~L}$. a Number density, b Temperature, c Mach number, d Flow streamlines 


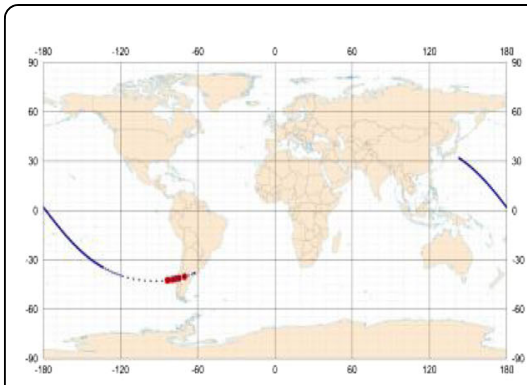

(a) Present numerical prediction

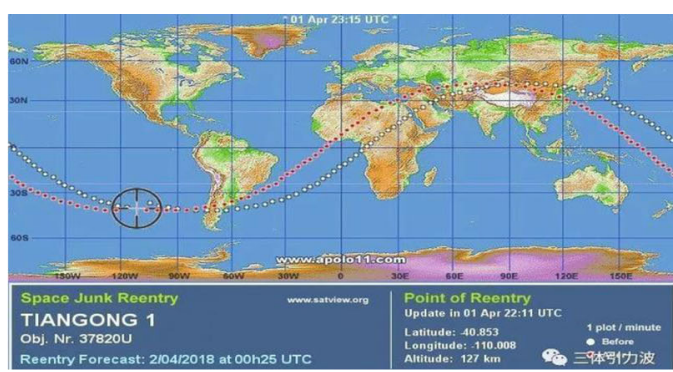

(b) Map calibration of NASA's post official website

Fig. 15 Validation of the present numerical forecast of uncontrolled reentry disintegration and the spacetracking results for falling area distribution of the Tiangong-1 spacecraft. a Present numerical prediction, $\mathbf{b}$ Map calibration of NASA's postofficial website

falling from outer space has been established [5-7, 9, 43, 44] with the unified simulation on ballistic trajectory, rapid engineering calculation modified by the present numerical algorithm results for aerothermodynamics covering various flow regimes, ablation, deformation failure and disintegration. Figure 15 shows the forecast of flight trajectory and falling area of disintegrated wreckage and debris from the Tiangong-1 spacecraft falling into the atmosphere with the comparison of the monitoring results afterwards from the map calibration of NASA-Tv's post website (www.apolo11.com) announcement in good agreement and compatibility. It is indicated from the falling reentry forecast that the uncontrolled Tiangong- 1 will be disintegrated firstly at the range of $110-105 \mathrm{~km}$, secondly at $100-95 \mathrm{~km}$, specially, the main bearing-cone platform and trajectory controlling engines will be disintegrated at $83 \mathrm{~km}-56 \mathrm{~km}$ and so on. The present numerical forecasting platform obtained the scope of falling area distribution of longitudinal length $1200 \mathrm{~km}$ and lateral width $100 \mathrm{~km}$ from the first disintegration to debris falling to the South $\mathrm{Pa}$ cific Ocean. These results on the multiple disintegration, falling area distribution and trajectory calculation coupled with aerothermodynamics for the uncontrolled Tiangong-1 spacecraft affirm the accuracy and reliability of the unified modeling and typical computation of structural deformation failure from thermodynamic response and hypersonic aerothermodynamics for falling disintegration along ballistic trajectory with different flying heights, Mach numbers covering various flow regimes from outer space to earth.

\section{Conclusion}

In this paper, the collision term of the Boltzmann equation is divided into elastic and inelastic collision terms. The inelastic collision is characterized into translational-rotational energy relaxation and translational-rotational-vibrational energy relaxation according to certain relaxation rates in real-time computation. Then, a kind of Boltzmann model equation considering the excitation of vibrational energy is constructed, and an implicit gas-kinetic unified algorithm has been presented to directly solve the unified Boltzmann model equation. The massively parallel MPI and OpenACC technique is built and applied to the irregular large-scale and multi-body reentry flows from free-molecular to continuum flow regimes. 
To simulate the disintegration of metal truss structure for spacecraft during uncontrolled falling back to the atmosphere, the dynamic thermo-mechanical coupling model has been derived by the variational principle on the basis of the dynamic thermoelasticity and the heat conductive equations, then the finite-element implicit schemes are constructed, as a result, the corresponding finite-element algorithm and computational procedures of FEA + GKUA have been established to simulate the transient thermal and mechanical damage behaviors of the structures under reentering aerothermodynamic environment as a new researching direction.

Integrated by developing the other simulation prediction methods as verification and supplement of reliable modeling for hypersonic reentry aerothermodynamics covering various flow regimes, the forecasting analysis platform of spacecraft falling flight track has been being established for the unified computation to reentry aerothermodynamics and structural deformation failure/ablation/disintegration, and has been applied to the numerical forecast of the flight track of the uncontrolled Tiangong-1 spacecraft, in which authenticates the correctness and validity of the computable modeling of Boltzmann-type velocity distribution function equation with internal energy excitation and the gas-kinetic massively parallel algorithm for hypersonic non-equilibrium aerothermodynamics during falling disintegration of the uncontrolled Tiangong-1 spacecraft.

As this work is only the beginning of numerical forecast for un-controlling spacecraft falling from outer space, further investigations on the three-dimensional irregular multi-body flows with real gas effects involving internal energy excitation around disintegrating debris of uncontrolled spacecraft, need to be studied in the future.

\section{Acknowledgements}

This work is supported by the National Key Basic Research and Development Program (2014CB744100), the National Outstanding Youth Fund(11325212), the Integrating Project of NSFC Great Researching Plan(91530319) of China, and the China Manned Space Engineering Office. The first author would like to thank his projects' group including Jie Liang, Zhonghua Li, Weibo Shi, Xinyu Jiang, Xuguo Li, Geshi Tang, Zhiyong Bai, Dun Li, Boqiang Du, Yu Xiao, Junlin Wu, Ming Fang, Siyao Su for their helpful work. Parts of present computation were carried out by the National Supercomputing Centers in Wuxi, Tianjin and Jinan. The authors are thankful to the reviewers for their valuable comments to improve the quality of the manuscript.

\section{Funding}

The National Key Basic Research and Development Program (2014CB744100), and the National Natural Science Foundation of China (91530319 and 11325212) support the present researches in the design of the study and collection, analysis, and interpretation of data and in writing the manuscript.

Availability of data and materials

Data supporting the results reported in the article can be found including where generated during the study. By data, the minimal dataset that would be necessary to interpret, replicate and build upon the findings reported in the article.

- The datasets generated and/or analysed during the current study are not publicly available due to the Chinese manned space engineering but are available from the corresponding author on reasonable request.

\section{Authors' contributions}

Z-HL designed the computable modeling of Boltzmann equation, the gas-kinetic unified algorithm for aerothermodynamics during falling disintegration of uncontrolled Tiangong-1 spacecraft, and computational procedures of FEA+GKUA to solve the transient thermal and mechanical damage behaviors of the structures. A-PP developed the gas-kinetic unified algorithm for the computable modeling of Boltzmann equation in solving the hypersonic non-equilibrium aerothermodynamics. QM designed the finite-element implicit schemes and computation of structural deformation failure to couple the dynamic thermoelasticity and the heat conductive equations. L-ND developed the rapid engineering calculation of ballistic trajectory combined with aerodynamics. X-WT designed the forecasting analysis platform of spacecraft falling flight track. X-ZS analyzed and collected to interpret the flying data during uncontrolled Tiangong-1 spacecraft falling back to the atmosphere. All authors read and approved the final manuscript. 


\section{Publisher's Note}

Springer Nature remains neutral with regard to jurisdictional claims in published maps and institutional affiliations.

\section{Author details}

${ }^{1}$ Hypervelocity Aerodynamics Institute, China Aerodynamics Research and Development Center, P.O.Box 211, Mianyang 621000, China. ${ }^{2}$ National Laboratory for Computational Fluid Dynamics, BUAA, No.37 Xueyuan Road, Beijing 100191, China. ${ }^{3}$ College of Mathematics, Sichuan University, Chengdu 610043, China.

Received: 1 November 2018 Accepted: 24 January 2019

Published online: 22 February 2019

\section{References}

1. Reyhanoglu M, Alvarado J (2013) Estimation of debris dispersion due to a space vehicle breakup during reentry. Acta Astronautica 86:211-218

2. Balakrishnan D, Kurian J (2014) Material thermal degradation under reentry aerodynamic heating. J Spacecr Rocket:1-10 https://doi.org/10.2514/1.A32712

3. Wu ZN, Hu RF, Qu X et al (2011) Space debris reentry analysis methods and tools. Chin J Aeronaut 24(4):387-395

4. Caggiano A, Etse G (2015) Coupled thermo-mechanical interface model for concrete failure analysis under high temperature. Comput Methods Appl Mech Eng 289:498-516

5. Li ZH, Ma Q, Cui JZ (2016) Finite element algorithm for dynamic thermoelasticity coupling problems and application to transient response of structure with strong aerothermodynamic environment. Commun Comput Phys 20(3):773-810

6. Peng AP, Li ZH, Wu JL, Jiang XY (2016) Implicit gas-kinetic unified algorithm based on multi-block docking grid for multi-body reentry flows covering all flow regimes. J Comput Phys 327:919-942

7. Li ZH, Peng AP, Wu JL, Ma Q, Tang XW, Liang J, Gas-Kinetic Unified Algorithm for Computable Modeling of Boltzmann Equation for Aerothermodynamics during Falling Disintegration of Tiangong-type Spacecraft, Proc. of $31^{\text {st }}$ Intern. Symposium on Rarefied Gas Dynamics, Glasgow, U.K., Jul.23-27, 2018

8. Bird GA (1994) Molecular gas dynamics and direct simulation of gas flows. Oxford University Press, Oxford

9. Liang J, Li ZH, Li XG, Shi WB (2018) Monte Carlo Simulation of Spacecraft Reentry Aerothermodynamics and Analysis for Ablating Disintegration. Commun Comput Phys 23(4):1037-1051

10. Cercignani C (1988) The Boltzmann equation and its applications. Springer-Verlag, New York

11. Wagner W (1992) A convergence proof for Bird's direct simulation Monte Carlo method for Boltzmann equation. J Stat Phys 66:1011-1044

12. Li ZH, Fang M, Jiang XY, Wu JL (2013) Convergence proof of the DSMC method and the gas-kinetic unified algorithm for the Boltzmann equation. Sci China-Phys Mech Astron 56(2):404-417

13. Bobylev AV, Rjasanow S (1999) Fast deterministic method of solving the Boltzmann equation for hard spheres. Eur J Mech B Fluids 18(5):869-887

14. Tipton EL, Tompson RV, Loyalka SK (2009) Chapmann-Enskog solutions to arbitrary order in Sonine polynomials II: viscosity in a binary, rigid-sphere, gas mixture. Eur J Mech B Fluids 28:335-352

15. Sone Y, Takata S, Ohwada T (1990) Numerical analysis of the plane Couette flow of a rarefied gas on the basis of the linearized Boltzmann equation for hard-sphere molecules. Eur J Mech B Fluids 9(3):449-456

16. Sheng Q, Tang GH, Gu XJ, Emerson DR, Zhang YH (2014) Simulation of thermal transpiration flow using a high-order moment method. Int J Mod Phys C 25(11):1450061

17. Tcheremissine FG (2006) Solution of the Boltzmann kinetic equation for high speed flows. Comput Math Math Phys 46 : 315-329

18. Bhatnagar PL, Gross EP, Krook M (1954) A Model Collision Processes in Gases: I. Small Amplitude Processes is Charged and Neutral One-Component System. Phys Rev 94:511-525

19. Holway LH Jr (1966) New statistical models for kinetic theory: methods of construction. Phys Fluids 9(9):1658-1673

20. Shakhov EM (1968) Generalization of the Krook kinetic relaxation equation. Fluid Dynamics 3(5):95-96

21. Rykov VA (1975) Model kinetic equation of a gas with rotational degrees of freedom. Fluid Dynamics 10:959-966

22. Yang JY, Huang JC (1995) Rarefied flow computations using nonlinear model Boltzmann equations. J Comput Phys 120: 323-339

23. Titarev VA, Shakhov EM (2002) Heat transfer and evaporation from a plane surface into a half-space upon a sudden increase in body temperature. Fluid Dynamics 37(1):126-137

24. Li ZH, Zhang HX (2003) Numerical investigation from rarefied flow to continuum by solving the Boltzmann model equation. Int J Numer Methods Fluids 42:361-382

25. Kudryavtsev AN, Shershnev AA (2013) A numerical method for simulation of microflows by solving directly kinetic equations with WENO schemes. J Sci Comput 57:42-73

26. Nie X, Doolen GD, Chen S (2002) Lattice Boltzmann simulation of fluid flows in MEMS. J Stat Phys 107:279

27. Meng JP, Zhang YH, Hadjiconstantinou NG, Radtke GA, Shan XW (2013) Lattice ellipsoidal statistical BGK model for thermal non-equilibrium flows. J Fluid Mech 718:347-370

28. Mandal JC, Deshpande SM (1994) Kinetic flux vector splitting for euler equations. Comput Fluids 23(2):447

29. Xu K (2001) A gas-kinetic BGK scheme for the Navier-stokes equations and its connection with artificial dissipation and Godunov method. J Comput Phys 171(1):289-335

30. Xu K, Li ZH (2004) Microchannel flow in the slip regime: gas-kinetic BGK-Burnett solutions. J Fluid Mech 513:87-110

31. Xu K, Huang JC (2010) A unified gas-kinetic scheme for continuum and rarefied flows. J Comput Phys 229:7747-7764

32. Liu S, Yu PB, Xu K, Zhong ZW (2014) Unified gas kinetic scheme for diatomic molecular simulations in all flow regimes. J Comput Phys 259:96-113

33. Yang LM, Shu C, Wu J (2014) A simple distribution function-based gas-kinetic scheme for simulation of viscous incompressible and compressible flows. J Comput Phys 274:611-632

34. Chen SZ, Xu K, Li ZH (2016) Gas kinetic scheme in Cartesian grid method for regular flows with different Mach numbers. J Comput Phys 326:862-877 
35. Li ZH, Zhang HX (2004) Study on gas kinetic unified algorithm for flows from rarefied transition to continuum. J Comput Phys 193:708-738

36. Li ZH, Zhang HX (2008) Gas-kinetic description of shock wave structures by solving Boltzmann model equation. Int J Comput Fluid Dyn 22(9):623-638

37. Li ZH, Zhang HX (2009) Gas-kinetic numerical studies of three-dimensional complex flows on spacecraft re-entry. J Comput Phys 228:1116-1138

38. Li ZH, Peng AP, Zhang HX, Yang JY (2015) Rarefied gas flow simulations using high-order gas-kinetic unified algorithms for Boltzmann model equations. Prog Aerosp Sci 74:81-113

39. Li ZH, Zhang HX (2007) Gas-kinetic numerical method solving mesoscopic velocity distribution function qeuation. Acta Mech Sinica 23(3):121-132

40. Li ZH, Zhang HX, Fu S (2005) Gas kinetic algorithm for flows in Poiseuille-like microchannels using Boltzmann model equation. Sci China-Phys Mech Astron 48(4):496-512

41. Gu W, Wang P (2014) A Crank-Nicolson difference scheme for solving a type of variable coefficient delay partial differential equations. J Appl Math ID560567:1-6

42. Hashiguchi K (2013) General description of elastoplastic deformation/sliding phenomena of solids in high accuracy and numerical efficiency: subloading surface concept. Arch Comput Methods Eng 20:361-417

43. Li ZH, Dang LN, Li ZH (2018) Study on N-S/DSMC hybrid numerical method with chemical non-equilibrium for hypersonic flow. Acta Aeronautica et Astronautica Sinica 39(10):122229

44. Li ZH. Technical Summary on Numerical Forecast and Hazard Analysis for Falling Disintegration of Uncontrolled Tiangong-No.1 Target Spacecraft, Tech. Rep. No. S2018.14, Hypersonic Aerodynamics Institute, China Aerodynamic Research and Development Center. 2018

45. Peng AP, Li ZH, Wu JL, Jiang XY (2017) Validation and Analysis of Gas-Kinetic Unified Algorithm for Solving Boltzmann Model Equation with Vibrational Energy Excitation. Acta Phys Sin 66(20):204704

\section{Submit your manuscript to a SpringerOpen ${ }^{\circ}$} journal and benefit from:

- Convenient online submission

- Rigorous peer review

- Open access: articles freely available online

High visibility within the field

- Retaining the copyright to your article

Submit your next manuscript at $>$ springeropen.com 